\title{
Editorial
}

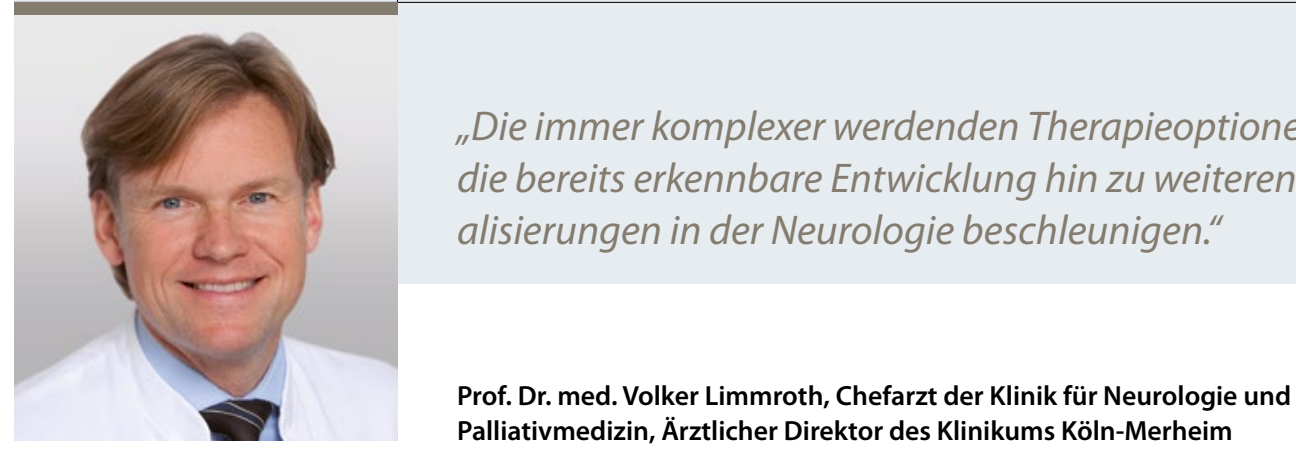

Palliativmedizin, Ärztlicher Direktor des Klinikums Köln-Merheim

\section{Neurologie 2013}

$\mathrm{F}$ ür uns Neurologen ist die Neurologie natürlich das spannendste Fach in der gesamten Medizin. Das Gehirn, das spannendste Organ, ohne das nichts geht. Alles andere kann man inzwischen austauschen, transplantieren oder irgendwie behandeln (fast). Unzählige Male bin ich gefragt worden, warum ich denn unbedingt in einem Fach wie der Neurologie arbeiten wolle, wo zwar vieles zu diagnostizieren, aber nichts zu behandeln sei. Das hat sich - wie wir wissen - gründlich geändert, ein Segen für alle Patienten und großartig für uns klinisch tätige Neurologen.

\section{Beschleunigter Wandel}

Im Jahr 2013 wird sich dieser Wandel weiter beschleunigen, und zwar in einem Maße wie selten zuvor. Allein für die Behandlung der Multiplen Sklerose werden 2013 aller Voraussicht nach gleich drei sehr verschiedene Substanzen zur immunologischen Therapie zugelassen: Fumarsäure (BG12), Teriflunomid und Alemtuzumab. Rekord: Drei neue Therapeutika in einem Jahr gab es bisher noch nie. Man braucht kein Prophet zu sein, um voraussagen zu können, dass diese Substanzen unsere derzeitigen therapeutische Algorithmen kräftig durcheinander wirbeln werden. Während Fumarsäure sehr wahrscheinlich als First-line-Therapie zugelassen werden wird und damit eine echte orale Alternative zu den bisherigen injizierbaren Immunmodulatoren darstellt, wird Alemtuzumab wohl als Eskalationstherapuetikum zugelassen. Beiden Therapeutika ist gemein, dass die Daten der Phase-III-Studien Wirksamkeitswerte erbrachten, die besser waren, als die der bisherigen Substanzen in der jeweiligen Therapiegruppe. Die Rolle von Teriflunomid ist schwerer vorherzusagen, aber als orale Therapie mit einer den Interferonen vergleichbaren Wirksamkeit wird es auch eine Alter- native zu den injizierbaren Basistherapeutika sein. Die Entwicklung ist mit der Zulassung dieser drei Substanzen aber noch lange nicht beendet. Die nächsten Substanzen, wie pegyliertes Interferon-beta 1a, monoklonale Antikörper gegen B-Zellen und IL2Rezeptoren oder auch selektivere S1P1-Modulatoren sind in Phase-III-Studien angekommen und werden in den nächsten zwei bis drei Jahren ebenfalls auf den Markt streben.

\section{Zeitgemäße Therapie der MS}

In dieser Ausgabe geht Professor Matthias Mäurer der Frage nach, inwieweit die derzeitige Eskalationstherapie in der Multiple Sklerose in Anbetracht dieser Entwicklungen noch zeitgemäß ist und ob Substanzen mit einer höheren Wirksamkeit, die als „Eskalationstherapeutika“ gelten, nicht bereits früher eingesetzt werden sollten. Diese Frage ist mehr als gerechtfertigt, denn die neuen Substanzen sind in ihrer Wirksamkeit den etablierten Immunmodulatoren zum Teil deutlich überlegen, dürfen aber als „second-line“ erst eingesetzt werden, wenn der Patient eine gewisse Krankheitsaktivität zeigt.

\section{Komplexere Substanzen und Therapiekonzepte}

Aber nicht nur in der Neuroimmunologie tut sich viel. In der Sekundärprävention des Schlaganfalls bei Patienten mit Vorhofflimmern werden nach Dabigatran und Rivaroxaban nun zwei weitere Substanzen auf den Markt kommen: Apixaban - gerade frisch zugelassen - und wahrscheinlich Ende des Jahres Edoxaban. Hier werden wir demnächst die Qual der Wahl haben und Abgrenzungskriterien wie Niereninsuffizienz, medikamentöse Interaktionen und andere erarbeiten müssen, um die Substanzen optimal nutzen zu können. 
Was aber bedeuten diese Neuerungen für uns Neurologen, abgesehen davon, dass wir neue Therapieoptionen haben? Es bedeutet, dass sowohl die Therapiekonzepte als auch die Substanzen selbst komplexer werden. Wir werden uns mehr mit den zum Teil hochspezifischen Nebenwirkungen, der Optimierung der Verträglichkeit und neuen Laborparametern vertraut machen müssen. Im Bereich der Neuroimmunologie werden wir uns auch mit weiteren Erkrankungen beschäftigen müssen, die bisher nicht zwangsläufig auf dem Radarschirm des Neurologen zu finden waren. Durch die Einführung von Natalizumab sind wir inzwischen mit der progressiven multifokalen Leukencephalopathie (PML) gut vertraut, durch Fingolimod haben wir Makulaödeme als mögliche Differenzialdiagnose bei Sehstörungen unserer MS-Patienten kennengelernt. Durch die Einführung von Alemtuzumab werden wir uns noch mit weiteren Autoimmunerkrankungen wie der Hashimoto-Thyreoiditis, der autoimmunen Thrombozytopenie (ITP) oder dem Goodpasture-Syndrom befassen müssen, um nur einige Beispiele zu nennen.

\section{Blick über den Tellerrand}

Auch der Blick über den neurologischen Tellerrand in die Kardiologie ist zunehmend hilfreich bis notwendig: Für die Erstgabe von Fingolimod ist es auch für einen Neurologen hilfreich einen AV-Block ersten Grades von einem AV-Block zweiten Grades unterscheiden zu können. Für die Sekundärprävention des Schlaganfalls ist es ferner sinnvoll, dass wir Neurologen sicher ein Vorhofflimmern im EKG erkennen, wissen wann elektrophysiologische Verfahren wie die Kardioversion (noch) sinnvoll sind und wann direkt mit einer oralen Antikoagulation begonnen werden sollte. Auch die verschiedenen klinischen Skalen, die nicht primär aus der Neurologie kommen, werden uns geläufiger werden müssen, da sie wichtige Entschei- dungskriterien bei der Indikationsstellung der neuen oralen Antikoagulanzien sein können. Natürlich kennen die meisten von uns den EDSS, aber wie gut kennen Sie sich mit vaskulären Risiko-Scores wie dem $\mathrm{CHADS}_{2}$ Score, dem $\mathrm{CHA}_{2} \mathrm{DS}_{2}$ VASc-Score oder dem HAS-BLEDScore aus?

\section{Subspezialisierungen}

Die zunehmend komplexer werdenden Therapieoptionen werden wahrscheinlich die bereits erkennbare Entwicklung hin zu weiteren Subspezialisierungen innerhalb der Neurologie beschleunigen. Das muss nicht notwendigerweise ein Nachteil sein. Die Neurologie ist weder ein rein diagnostisches Fach, noch ist sie ein kleines Nebenfach - sie hat sich zu einem großen therapeutischen Fach entwickelt. Weder in der Praxis noch in der Klinik werden wir als einzelne alle Bereiche der Neurologie abdecken können. Das ist keine Schande, sondern eine Entwicklung, die andere große Fächer wie die Innere Medizin schon hinter sich haben. Wir können und sollten uns also freuen : Neurologie - ein zunehmend größer werdendes therapeutisches Fach, attraktiv wie nie zuvor!

Mit kollegialen Grüßen,

Ihr

Volker Limmroth

Korrespondenz:

Prof. Dr. med. Volker Limmroth

Chefarzt der Klinik für Neurologie und Palliativmedizin

Klinikum Köln-Merheim

Ostmerheimer Str. 200, 51109 Köln

E-Mail: LimmrothV@kliniken-koeln.de

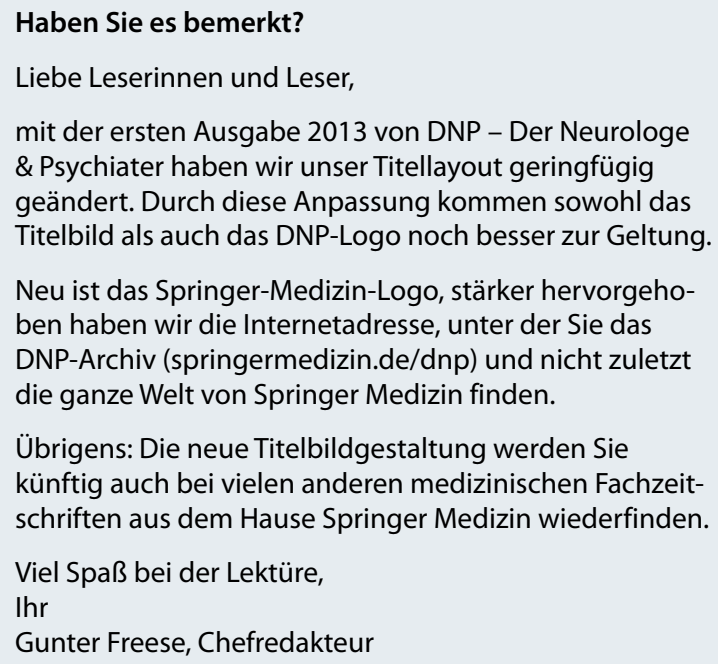

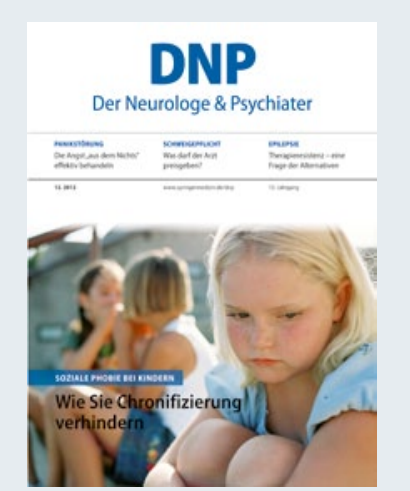

„Aus alt ...

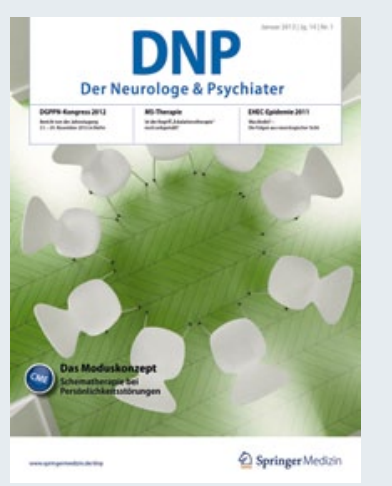

... mach neu!" 